\title{
非人灵长类位置行为的辐射适应与研究进展
}

\author{
张 晶 ${ }^{1}$, 齐晓光 ${ }^{1, *}$, 张 堪 ${ }^{1}$, 张 培 ${ }^{1}$, 郭松涛 ${ }^{1}$, 魏 玮 ${ }^{1}$, 李保国 ${ }^{1,2, *}$
}

(1. 西北大学 生命科学学院, 教育部西部资源生物与现代生物技术重点实验室, 西安 710069; 2. 陕西省科学院动物研究所, 西安 710032)

摘要：野生动物在长期进化中形成特定的行为模式以适应特定的生存环境。灵长类的位置行为研究对于理解 灵长类进化、适应性的多样化以及生态学和解剖学十分关键。该文对已有文献进行了综述, 针对位置行为的分类、 季节性、以及具体地点和年龄性别差异性进行了总结, 分析了灵长类位置行为的发展、特征以及研究现状, 以期 对今后非人灵长类位置行为的研究提供借鉴和参考, 从而促进我国灵长类行为生态学理论体系的发展。

关键词: 非人灵长类; 位置行为; 移动; 姿势

中图分类号：Q959.848 文献标志码：A＼cjkstart文章编号：0254-5853-(2012)05-0511-12

\section{Diversity and development of positional behavior in non-human primates}

\author{
ZHANG Jing ${ }^{1}$, QI Xiao-Guang ${ }^{1,}$, , ZHANG Kan ${ }^{1}$, ZHANG Pei ${ }^{1}$, GUO Song-Tao ${ }^{1}$, \\ WEI Wei ${ }^{1}$, LI Bao-Guo ${ }^{1,2, *}$ \\ (1. College of Life Sciences, Northwest University, Key Laboratory of Resource Biology and Biotechnology in Western China of Ministry of \\ Education, Xi'an 710069, China; 2. Institute of Zoology, Shaanxi Academy of Sciences, Xi'an 710032, China)
}

\begin{abstract}
In long-term evolution, wildlife in general and primates in particular have formed specific patterns of behavior to adapt to a diverse variety of habitat environments. Current research on positional behavior in non-human primates has been found to explain a great deal about primate adaptability diversification, ecology, anatomy and evolution. Here, we summarize the noted classifications and differences in seasonal, site-specific and sex-age positional behaviors while also reviewing the development and status of non-human primate positional behavior research. This review is intended to provide reference for the future research of non-human primates and aid in further research on behavioral ecology of primates.
\end{abstract}

Key words: Nonhuman primates; Positional behavior; Locomotor behavior; Postural behavior

野生动物在长期进化中形成特定的行为模式 以适应特定的生存环境(Jiang, 2004)。这些行为模式 不仅取决于个体体型大小和解剖学特征, 还取决于 非生物环境(如温度、降雨)和生物环境(栖息地结 构、物种丰富度、资源分布及可获得性)的空间差异 (Garber, 2007; Prates \& Bicca-Marques, 2008)。因此, 开展动物行为模式研究将有助于了解动物对其栖 息生境的行为适应机制(Jiang, 2004; Garber, 2007)。

在早先的树栖生活环境中, 灵长类表现成功的 辐射型进化。其种间甚至种内运动模式十分多样且 存在极大差异, 这些差异体现在种间或个体间为获
取食物资源而开发利用环境的途径(Niemitz，1984; Cant，1986)、躲避天敌、以及移动时的能量消耗等 方面(Oxnard et al, 1990; Hunt, 1991; Garber, 1992; Anemone, 1993)。由于灵长类世系开采生活环境能 力的重要进化与位置行为和位置生态学相关, 因此, 灵长类位置行为研究对于理解灵长类适应性的多 样化非常关键(Garber, 2007), 而位置行为研究的主 要目的则是理解种群与自然环境的相互作用以及 适应的进化性关系。

目前, 已有较多学者关注此方面的研究及成果, 国外关于非人灵长类位置行为的研究也已展开, 但

收稿日期：2012-03-20; 接受日期：2012-06-01

基金项目：国家自然科学基金 (31130061, 30900168, 30970444); 国家大学生创新性实验计划项目(101069711)

*通信作者(Corresponding authors), E-mail: qixg@nwu.edu.cn; baoguoli@nwu.edu.cn

第一作者简介: 张晶, 女, 硕士; 主要从事灵长类行为生态学研究; E-mail: youha0816@sina.com 
国内还鲜有报道。本文对已有文献进行了综述, 分 析灵长类位置行为的分类、发展、特征以及研究现 状, 以期对今后非人灵长类位置行为的研究提供借 鉴和参考。

\section{1 位置行为概述}

\section{1 位置行为的定义}

位置行为 (positional behavior) 是动物为了适应 不同生活环境而采取的不同生存应对策略。灵长类 位置行为对其活动和环境的具体情况有具体的针 对性。也就是说, 某一位置行为(进食 vs 移动 vs 休 息)在特定活动中或在某一特定直径、方向和承重能 力的支撑物上会发生的更为频繁 (Ripley，1967; Kinzey, 1967; Garber, 1980, 1998; Bergeson, 1998)。

\section{2 位置行为的分类}

位置行为研究是灵长类肢体运动模式研究的 一种更为准确的说法(Prost, 1965)。其研究内容主要 包括两部分: 姿势行为 (postural behavior)和移动行 为(locomotor behavior)(Garber, 1984)。姿势行为即当 一个个体的重心保持相对稳定，四肢部分抓握适当 可发生相对移动的一系列目的性行为(Prost, 1965)。 姿势行为在进食和休息中较常见(坐、躺、四脚站立、 抓握、通过两个或三个肢体悬挂等)。而移动行为则 是指个体的重心发生相对移动时四肢的运动情况, 常与个体移动、受食和躲避天敌等行为有关。移动 是一种达到目的的手段, 例如, 为寻找食物或休息 地而移动, 为逃离捕食者而快速跑跳, 或进行社交 行为等。奔跑、攀爬和跳跃都是常见的移动 行为。

Hunt et al (1996)对灵长类的位置行为模式进行 了标准化定义。他们认为不一致的传统术语和不正 确的模式分类阻碍了各研究间的互相比较。一些研 究者未按照定义使用常用词汇, 而是在记录中使用 自己针对某物种而定义的词汇，从而阻碍了非人灵 长类位置行为的对比研究。还有一些研究人员创造 新词以适用于其研究的物种, 但却无法将其应用于 其他物种的位置行为模式, 且此类新词与已有的标 准定义可能会部分重叠、完全涵盖或混淆, 从而使 得原本清晰的定义变得混乱。许多学者认为, 目前 有 3 个方面的行为方式的区分很重要：(1) 肱骨外 展的行为应有别于肱骨内收的行为; (2) 坚直方向 的移动应有别于水平方向的移动; (3) 悬吊行为应 区别于有支撑物的行为。
Hunt et al (1996)形象地定义了 32 种灵长类位 置行为, 其中包括 14 类姿势行为和 18 类移动行为: 1.2.1 姿势行为(定义框架来源于 Hunt et al, 1996) ZS1 (坐, sit): 由坐骨承担一半以上体重。包括: ZS1-a (静坐, sit-in), 即由坐骨和脚共同承重, 脊椎 弯曲(Fleagle, 1978), 躯干直立; ZS1-b (久坐, sit-out), 与 ZS1-a 的不同在于脚仅用于维持平衡而不承重, 后肢伸展, 脚伸向外; ZS1-c (后肢撑坐, foot-prop sit)，与 ZS1-a 相似，后肢由脚支撑，身自由前肢支 撑; ZS1-d (坐骨正坐, ischium-sit), 即仅由坐骨(包 括坐胼胝)承重; ZS1-e (座椅正坐, chair-sit), 即躯干 直立，坐骨和背部承担大部分体重，如同人类坐在 椅子上，故称为座椅正坐; ZS1-f (坐/前肢悬挂, sit/forelimb-suspend), 即由前肢抓附支持物以稳定 身体并承担一部分体重(图 1a,b)(Hunt et al, 1996)。

ZS2 (蹲, squat): 体重靠脚或脚、髋关节和膝关 节的同时强烈弯曲来支撑，前肢和坐骨不承重，背 部弯曲(Hunt et al, 1996)。

ZS3 (抓握依附, cling): 该曲肢姿势常见于垂直 或接近垂直的支撑物。包括：ZS3-a（双手依附, bimanual cling), 即垂直依附(vertical cling), 通常肘 部、膝关节和髋关节均弯曲, 前肢内收抱住支撑物, 躯干笔直或弯曲，后肢至少承受一半的体重(图 1c), 坐骨不承重; ZS3-b (单手依附, unimanual cling), 与 双手依附类似，但仅由一只前肢抓住支持物，另一 前肢弯曲； ZS3-c（依附／前肢悬挂， cling/forelimb-suspend), 与单手依附不同的是, 未 抓住支持物的前肢以悬臂方式伸张(Hunt et al, 1996)。

ZS4 (站立, stand), 常见姿势包括: ZS4-a (四足 站立，quadrupedal stand), 四肢站在水平支撑物上， 肘部和膝盖相对伸展, 身区干近乎水平(图 1d); ZS4-b (三足站立，tripedal stand)，与四足站立类似，但仅 由两后肢和其中一前肢承重(如：狒狒，图 1e); ZS4-c (蜷缩, crouch); ZS4-c-1(完全蜷缩), 肘部和膝 盖弯曲; ZS4-c2 (前肢蜷缩), 肘部弯曲, 膝盖不弯曲; ZS4-c-3(后肢蜷缩), 膝盖弯曲, 肘部不弯曲(Hunt et al, 1996)。

ZS5 (双足站立, bipedal stand), 包括: ZS5-a (双 足弯曲站立, flexed bipedal stand), 在身体其他部位 无明显支撑的情况下使用后肢站立，身干通常弯 曲 $45^{\circ}$, 髋关节和膝关节均弯曲(图 1f,g); ZS5-b (双足伸展站立, extended bipedal stand), 与人类的 
双足站立类似，髋关节和膝关节完全伸展且前肢无 明显支撑作用，躯干直立; ZS5-c (站立/前肢悬挂, stand/forelimb-suspend), 前肢承担一半以上的体重, 后肢也有明显的支撑作用(Hunt et al, 1996)。

ZS6 (三角支撑, tripod), 包括两种方式: ZS6-a (水平三角支撑, horizontal tripod), 即尾巴悬挂于支 撑物上与双足共同承重, 躯干水平或近似水平 (Fontaine，1990); ZS6-b（垂直三角支撑，vertical tripod), 双足弯曲站立, 尾巴保持僵硬并与地面有 力接触以维持身体稳定(Hunt et al, 1996)。

ZS7 (悬臂, cantilever): 双脚固定下半身以保持 平衡, 身体与支撑物近乎垂直, 躯干伸直, 并可伸 出一只前肢捕获食物(图 1h)。包括: ZS7-a (水平悬 臂, extended cantilever), 膝关节与身体伸展, 常见 于懒猴科和鼠狐猴科动物; ZS7-b (倾斜悬臂, lean out cantilever), 膝关节弯曲(如: 眼镜猴) (Hunt et al, 1996)。

ZS8 (前肢悬挂, forelimb-suspend, arm-hang): 即前肢抓握位于其重心上方的支持物且承担一半 以上的体重。包括: ZS8-a (单臂悬挂, unimanual forelimb-suspend), 即其中一只前肢抓握悬挂, 肱骨 弯曲, 肘部完全伸展, 躯干垂直, 身体其他部分可 能支撑少量体重(图 1i); ZS8-b (双臂悬挂, bimanual forelimb-suspend): 两前肢抓握悬挂(YD12, 但尾巴 不支撑身体); ZS8-c (前肢悬挂 / 坐， forelimb-suspend/sit), 与坐/前肢悬挂类似, 但由前 肢(而非坐骨)承担一半以上体重; ZS8-d (前肢悬挂/ 蹲, forelimb-suspend/ squat), 前肢抓握支撑物使上 半身悬挂的蹲坐形态; ZS8-e（前肢悬挂/站立, forelimb-suspend/stand), 前肢抓握悬挂且双足站立 承担部分体重，身干倾斜 $\geqslant 45^{\circ} ; \mathrm{ZS} 8-\mathrm{f}$ (前肢悬挂/跃， forelimb-suspend/cling), 前肢抓握支撑物, 后肢弯 曲并承担近一半的体重; ZS8-g (前肢悬挂/躺, forelimb-suspend/lie), 上半部分身体悬挂的躺姿; ZS8-h (躯干垂直悬挂, trunk-vertical-suspend), 前、 后肢任意组合抓握悬挂以支撑身体，四肢紧绷，身区 干直立(如，猩猩) (Hunt et al, 1996)。

ZS9 (后肢悬挂, hindlimb-suspend): 即使用一只 或两只后肢悬挂。包括: ZS9-a (后肢弯曲悬挂, flexed-hindlimb-suspend), 膝关节和/或髂关节弯曲; ZS9-b (后肢伸展悬挂 extended-hindlimb-suspend), 膝关节和髋关节均伸展(Hunt et al, 1996)。

ZS10 (前后肢悬挂 forelimb-hindlimb-suspend, arm-foot hang)：即使用任一前肢和任一后肢悬挂， 四肢伸展，与前肢悬挂不同的是，躯干处于近乎水 平方向(如: 猩猩，图 1j)。包括: ZS10-a (身体同侧 的前后肢悬挂, ipsilateral forelimb-hindlimbsuspend), 即用身体同一侧的前肢和后肢抓握悬挂, 躯干水平; ZS10-b（身体对侧的前后肢悬挂， contralateral forelimb-hindlimb-suspend), 即用任一 前肢和对侧后肢抓握悬挂, 躯干水平(Hunt et al, 1996)。

ZS11 (四肢悬挂, quadrumanous-suspend): 即躯 干水平悬挂，四肢所提供的支撑力几乎相等(Hunt et al, 1996)。

ZS12 (尾巴悬挂 tail-suspend), 包括: ZS12-a (尾 巴悬挂, tail-suspend), 即由尾巴承担大部分体重, 四肢几乎不承重; ZS12-b (尾巴/前肢悬挂, tail/forelimb-suspend), 尾巴承担至少一半的体重, 前肢也有承重, 肱骨外展, 肘部完全伸展(图 1k); ZS12-c (尾巴/后肢悬挂, tail/hindlimb-suspend), 体 重基本由后肢和尾巴支撑; ZS12-d (直立行走尾巴/ 四肢悬挂，orthograde tail/quadrumanous-suspend), 四肢和尾巴均承重, 身干垂直; ZS12-e（俯身行走尾巴/四肢悬挂, pronograde tail/quadrumanoussuspend)，四肢和尾巴均承重，躯干水平(Hunt et al, 1996)。

ZS13 (躺, lie): 即在一个近乎水平的支撑物上, 躯干保持伸直并承担大部分体重，侧躺时个体可能 会用肘部来支撑身体。主要方式包括: ZS13-a (仰 躺, supine lie), 四肢可能会在身下弯曲, 也可能会 承受一些重量, 腹部和手或脚所承担的重量几乎相 同(Fontaine，1990); ZS13-b (四肢伸展躺，sprawl), 通常发生在个体在树枝上以腹部支撑着休息时，四 肢自然垂悬, 有时是为了凉爽(图 1 1; Fontaine, 1990); ZS13-c (背躺, back lie), 主要依靠背部支撑 体重; ZS13-d (侧躺, lateral lie), 体重主要靠单侧身 体支撑; ZS13-e (坐/躺, sit/lie): 坐着休息, 身体上半 部分由肘部支撑(Hunt et al, 1996)。

ZS14 (架桥姿势, postural bridge): 即由后肢抓 住河流、树枝等间隙一边的支持物, 前肢抓住另一 边的支持物，以使身体跨过间隙，母猴有时会将其 身体作为紧猴渡过间隙的桥梁(Hunt et al, 1996)。

1.2.2 移动行为(定义框架来源于 Hunt et al, 1996)

YD1 (四肢行走, quadrupedal walk): 即在倾角 $<45^{\circ}$ 的支持物上移动, 身体匍匐且与支撑物近似平 


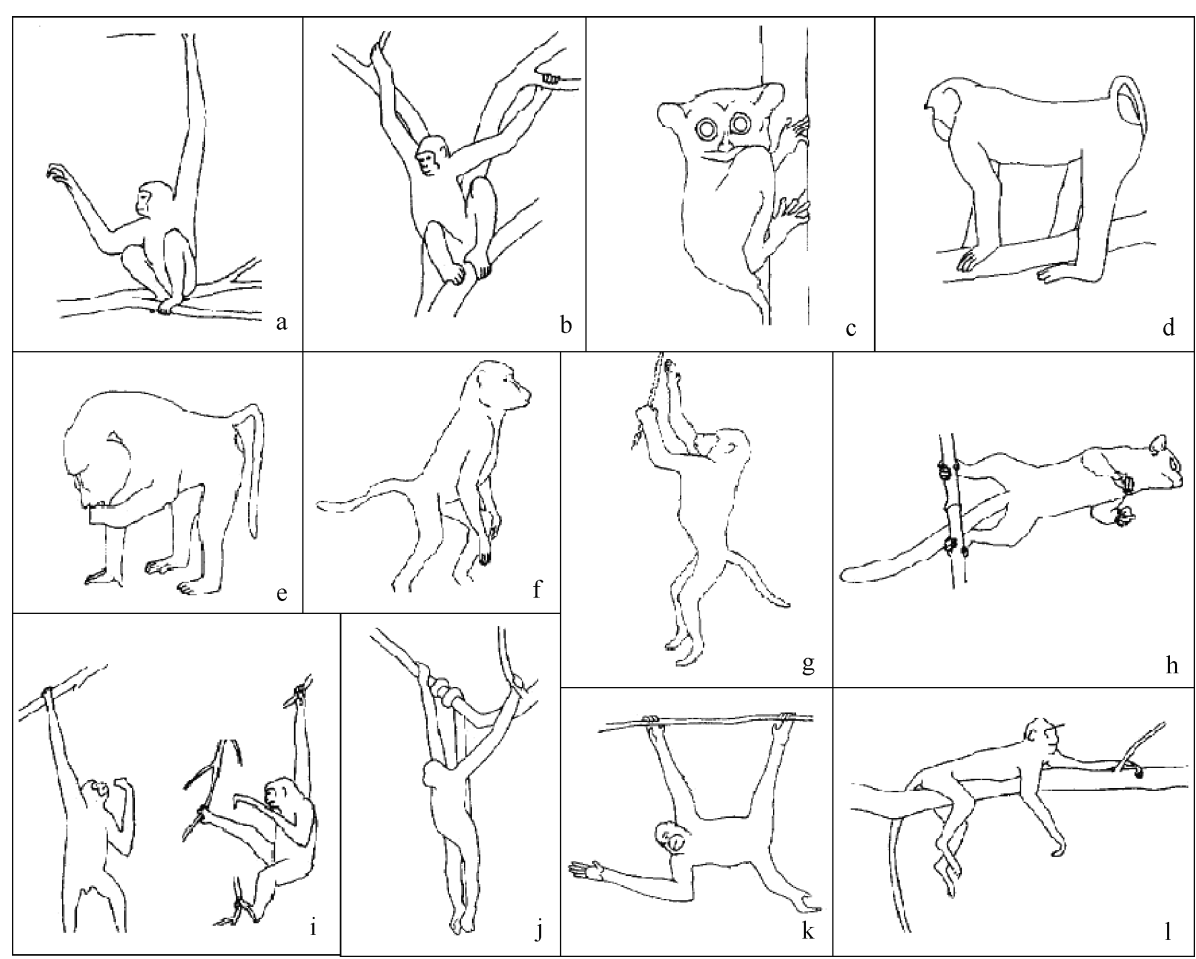

图 1 姿势行为 (Hunt et al, 1996)

Fig. 1 Postural behavior (Hunt et al, 1996)

a, b) 坐/前肢悬挂(sit/forelimb-suspend); c) 双手依附(bimanual cling); d) 四足站立(quadrupedal stand); e) 三足站立(tripedal stand); f, g) 双足弯曲站立 (flexed bipedal stand); h) 悬臂 (cantilever); i) 单臂悬挂(unimanual forelimb-suspend); j) 尾巴/前肢悬挂(tail/forelimb-suspend); k) 前后肢悬挂 (forelimb-hindlimb-suspend); 1) 四肢伸展躺(sprawl)。

行，四肢与支撑物有序接触，行走与奔跑的主要区 别在于行走速度较慢。包括: YD1-a (对称步态行走, symmetrical gait walk), 分为行走和蹲伏行走，前者 通常是四肢以对角线顺序与支撑物接触，四肢伸 直，后者与前者相似，但肘部和膝盖弯曲，以使身 体更靠近支撑物而更加稳定(图 2a); YD1-b (非对称 步态行走, asymmetrical gait walk); YD1-c (不规则 步态行走, irregular gait walking)。其中 YD1-b 可具 体分为以下几类: YD1-b-1 (跳跃), 前、后肢同时向 前移动，身体同侧肢体同时抓住支撑物; YD1-b-2 (三肢跳跃)，与 YD1-b-1 类似，但仅使用一只前肢; YD1-b-3( 慢跳), 与 YD1-b-1 类似, 速度低; YD1-b-4(倚靠行走), 躯干垂直前进(在陆地上), 后 肢移动时躯干在两前肢间来回交替, 且几乎只出现 于跳跃的急剧降落阶段; YD1-b-5( 经轴跳 跃,Transaxial bound); 前、后肢同时接触支持物, 步 长相当长且背部伸展, 移动时后肢会跨过前肢; YD1-b-6(不完全跳跃), 只有一组肢体(前肢或后肢) 同步移动。YD1-c 可具体分为以下几类: YD1-c-1(攀 爬), 即匍匐攀爬, 步态无规律; YD1-c-2 (缓步行走, tardigrady), 缓慢的匍匐前进, 每次只移动其中一
只四肢，且这种移动行为包括许多不规则肢体移动 (Hunt et al, 1996)。

YD2 (三肢行走, tripedal walk): 与四肢行走类 似，但移动时仅使用三个肢体，剩下的一个肢体通 常用于搬运物体。包括: YD2-a (前肢式三肢行进), 两前肢用于行进，一个后肢用于搬运物体； YD2-b(后肢式三肢行进), 两后肢用于行进, 一个 前肢用于搬运物体(Hunt et al, 1996)。

YD3 (两足行走, bipedal walk), 包括: YD3-a (伸展式两足行进, extended bipedal walk), 后肢提供 支持力和推进力, 臀部和膝盖均伸展, 与人类行走 类似; YD3-b (弯曲式两足行进, flexed bipedal walk), 与 YD3-a 类似, 但臀部和膝盖相对较弯曲(图 2b) (Hunt et al, 1996)。

YD4 (四肢奔跑, quadrupedal run): 利用非对称 或不均匀步态迅速移动, 有一个自由飞跃的阶段。 包括: YD4-a (非对称式奔跑, asymmetrical gait run), 多为急速的跳跃和不完全跳跃; YD4-b (不规则式奔 跑, irregular gait run), 步法无规律的快速移动(Hunt et al, 1996)。

YD5 (三肢奔跑, tripedal run)：仅其中三个肢体 
与地面接触的急速移动(Hunt et al, 1996)。

YD6 (两足奔跑, bipedal run): 该过程中包含一 个自由飞跃的阶段, 而大多数灵长类的 “跑” 实际 上是快速的行走，无飞跃阶段(Hunt et al, 1996)。

YD7 (两足跳跃, bipedal hop): 两后肢几乎同时 蹬起着地, 包含一个自由飞跃的阶段(身体的任何 部分均不接触支撑物)，身干直立(Hunt et al, 1996)。

YD8 (垂直攀爬, vertical climb), 包括: YD8-a (垂直攀爬, vertical scramble), 在各种角度的树枝上 攀爬, 支撑物向上 $>45^{\circ}$ 倾角均可认为是垂直攀爬; YD8-b（曲肘式垂直攀爬, flexed-elbow vertical climb), 在倾角 $\geqslant 45^{\circ}$ 的树枝上攀登, 四肢的移动遵 循对角线顺序, 身体匍匐几乎与正在攀爬的树枝平 行(图 2c); YD8-c (伸肘式垂直攀爬, extended-elbow vertical climbing), 在倾角 $>45^{\circ}$ 的大型支撑物上(对 于黑猩猩和狒狒来说直径 $>20 \mathrm{~cm})$ 攀爬, 与垂直和 梯式攀爬相似，但肘部伸展(图 2d); YD8-d (梯式攀 爬, ladder climb), 与曲肘式攀爬相似, 单支撑物通 常是相对水平的, 该行为与人以对角线式的步法爬 梯子类似; YD8-e (跳跃攀爬, pulse climb), 即垂直 跳跃(vertical bound), 在倾角 $>45^{\circ}$ 的树枝上移动, 前 肢抓住支撑物，后肢收缩在身下，后肢和背部的伸 展所产生的推进力促使身体向上，同时前肢放开支 撑物去抓取另一更高的支撑物; YD8-f (前肢提升, bimanual pull-up): 即牵引或提升(hauling or hoisting), 前肢抓住一水平支持物, 以肘的收缩和伸展来提升 身体, 脊柱弯曲以便将后肢牵引到支撑物上; YD8-g (头部优先下降, head-first descent), (该姿态 可具体分为: YD8-g-1(对称式头部优先下降), 与四 肢行走类似，且前肢在急剧的下降过程中有制动的 作用(可能出现滑行); YD8-g-2(非对称式头部优先 下降), 在一些倾角、粗细各异的树枝间下降(与对称 式相似), 步法不对称; YD8-g-3(瀑布式下落), 与 YD8-g-2 相似, 但作为支撑物的树枝较小且纵横交 错, 四肢和尾巴在下降过程中起连续制动作用); YD8-h（头部优先跳跃式下降, head-first bounding descent): 两前肢首先一起移动, 两后肢随后一起移 动, 四肢均有制动作用; YD8-i (尾部优先下降, rump-first descent), [该姿态可具体分为: YD8-i-1(对 称式尾部优先下降), 在倾角 $>45^{\circ}$ 的支撑物上四肢 垂直下降(相当于与向上攀爬相反); YD8-i-2(非对称 式尾部优先下降)，在方向、粗细各异的树枝间下降]; YD8-j (侧面垂直下降, sideways vertical descent), 身
体与支撑物的轴线垂直、处于身体下方的前后肢提 供主要的制动力; YD8-k (匍匐滑动, pronograde slide), 头部在前, 四肢紧握支撑物, 在光滑、倾斜 的树枝上被动下降，身体通过前、后肢及其他部位 与支撑物之间的滑行而移动, 身体匍匐并与支持物 保持平行; YD8-1 (摩擦支撑滑行, fire-pole slide), 慰 部在前, 在较粗的(直径 $>20 \mathrm{~cm}$ )垂直或近似垂直的 支撑物上被动滑行, 躯干直立，四肢环抱支撑物， 依靠身体其他部分的微小滑动来下降, 并可通过前 肢来调整下降速率(Hunt et al, 1996)。

YD9 (平行悬吊式移动, torso pronograde suspensory locomotion), 包括: YD9-a (四肢倒挂行 走, inverted quadrupedal walk), 四肢以各种组合并 用，身区匍匐，四肢紧张，通常步法有序; YD9-b (四肢倒挂奔跑, inverted quadrupedal run), 同 YD9-a, 但速度较快; YD9-c (倒挂式攀爬, inverted scramble), 同 YD9-a(在不规则倾角与大小的支持物上) (Hunt et al, 1996)。

YD10 (垂直悬吊式移动, torso-orthograde suspensory locomotion), 包括: YD10-a (十字式臂荡 前进, brachiate), 一只前肢接替另一前肢的直立悬 吊式移动，该姿态在灵长类中十分常见，前肢承受 大部分体重，后肢与尾巴有时也会承担部分体重， 身体有较大的旋转角度 $\left(\sim 180^{\circ}\right)$, 肱骨外展且肘部伸 展，在新大陆猴中该行为需要尾巴的协助(图 2e); YD10-b (十字式跳跃前进, brachiating leap), 借助于 迅速十字式前进的惯性，单臂摇摆推进身体向前; YD10-c (十字式弹跳前进, ricochetal brachiation), 一只前肢接替另一前肢的直立悬吊移动, 包含一个 自由飞跃的过程, 相对十字式臂荡前进更为迅速, 该模式需要尾巴协助; YD10-d (前肢摇摆, forelimb swing, arm swing), 与十字式前进类似, 但伴随些许 身体的旋转, 在新大陆猴中通常需要尾巴缠绕协助; YD10-e (屈肘式前臂摇摆, flexed-elbow forelimb swing), 与 YD10-d 相似, 但肘部弯曲; YD10-f (转移, transfer), 该移动行为通常由两前肢的悬挂开始, 包 括一个十字式前进的间隙结束动作 (一个跃进), 通 过前肢的不断转换将身体逐渐过渡到邻近的支撑 物上, 躯干保持一定程度的坚直, 在新大陆猴中通 常需要尾巴协助; YD10-g (直立攀爬, orthograde clamber, cautious climbing, amoebic suspensory locomotion), 前肢悬挂的水平前进, 躯干垂直, 后 肢提供协助, 四肢均有推进作用, 向外伸展的前肢 
承受大部分体重；YD10-h (遏制式下降，arrested drop), 从一个支撑物顶端向下移动, 以坐着或外展 的前肢、伸展的肘部而支撑的身体发起，躯体保持 直立下降, 个体移动到支撑物下端时慰部先着地 (Hunt et al, 1996)。

YD11 (桥渡, bridge), 包括: YD11-a (匍匐桥渡, cautious pronograde bridge), 躯体匍匐, 小心跨过障 碍物间隙的移动方式; YD11-b (跃进式桥渡, lunging bridge)，脚掌或尾巴抓住支持物然后以一个跃进跨 过障碍物间隙, 以使前肢抓住另一个较远的支撑物 (图 2f); YD11-c (悬挂式桥渡, supinograde bridge), 同 YD11-b, 同时悬挂; YD11-d (垂直向上桥渡, upward vertical bridge), 同 YD11-b, 但前进方向的 角度 $\geqslant 45^{\circ}$; YD11-e (下降式桥渡, descending bridge), 由后肢悬挂开始的不完整跳跃，前进方向的角度 $\leqslant$ $45^{\circ}$ (Hunt et al, 1996)。

YD12 (猛冲, scoot): 身体依靠滑动产生的力向 前, 坐骨承担大部分体重, 当身体被推进向前时膝 盖和大腿仍保持弯曲(Hunt et al, 1996)。

YD13 (飞跃 leap), 一种跨越横沟的运动模式, 后肢提供主要推进力, 包含躯体伸展的自由飞跃阶 段(与弹跳不同)。包括：YD13-a (匍匐飞跃, pronograde leap)，躯体在起跳时处于匍司状态; YD13-b (原式飞跃, pumping leap)，与匍匐飞跃类似， 加强树枝上的摇摆动作, 以此来增加飞跃 距离; YD13-c（前后肢悬挂式飞跃， hindlimb-forelimb suspensory leap), 前、后肢任意组 合悬挂, 并在起跳时提供推进力; YD13-d (后肢悬 挂式飞跃, hindlimb suspensory leap), 由一个或两个 后肢悬挂开始的飞跃，前肢前伸; YD13-e (垂直依 附型飞跃, vertical clinging leap), 起始动作为躯体 直立依附于一个相对垂直的支撑物, 后肢提供推进 力, 与匍匐飞跃的区别在于起始的依附(图 2g) (Hunt et al, 1996)。

YD14 (下落, drop): 与飞跃的区别在于起跳不 是由肌肉的推进作用开始, 而是在松开一个支撑物 后降落时发起的。包括：双足下落, 四肢下落, 单 手下落, 前肢悬挂式下落, 屈肘式悬挂下落, 尾吊 式下落, 后肢悬挂式下落和前后肢悬挂式下落等 (Hunt et al, 1996)。

YD15 (摇尾, tail swing): 尾巴悬挂时的摆动推 动动物跨过间隙(Hunt et al, 1996)。

YD16 (飞跃、下落、摇尾时的着陆, landings for leap, drop, and tail swing), 包括: YD16-a (前肢落地,

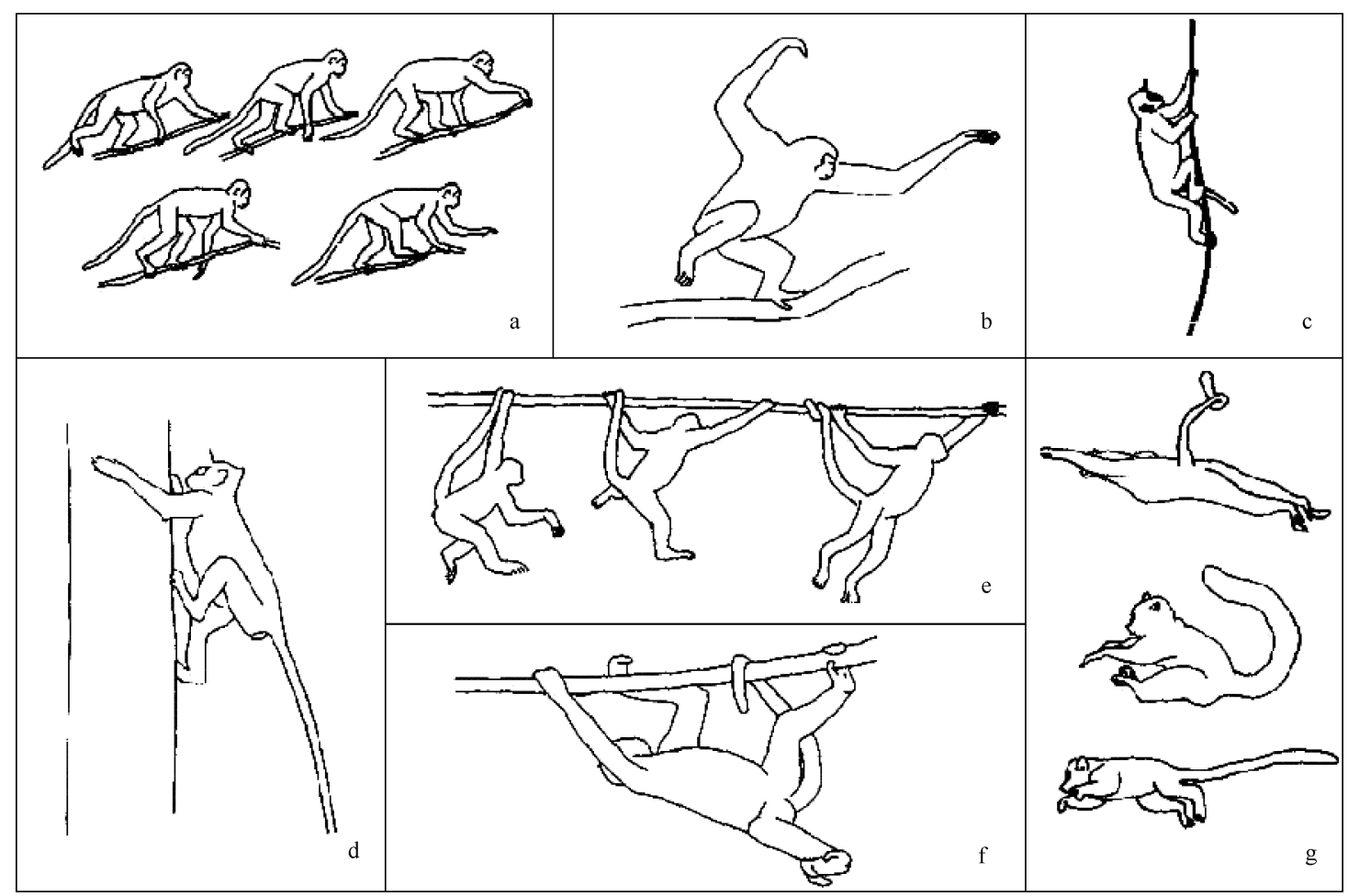

图 2 移动行为 (Hunt et al, 1996)

Fig. 2 Locomotor behavior (Hunt et al, 1996)

a) 对称步态行走(asymmetrical gait walk); b) 弯曲式两足行进(flexed bipedal walk); c) 曲肘式垂直攀爬(flexed-elbow vertical climb); d) 伸肘式垂直攀爬 (extended-elbow vertical climbing); e) 十字式臂荡前进(brachiate); f) 跃进式桥渡(lunging bridge); g) 垂直依附型飞跃(vertical clinging leap)。 
forelimb landing), 前肢起主要的缓冲作用，身体始 终保持相对匍匐; YD16-b (前肢悬挂落地, suspensory forelimb landing), 通常伴随前肢悬挂; YD16-c（前后肢悬挂落地, hindlimb-forelimb suspensory), 前后肢(主要为前肢)任意组合完成缓 冲; YD16-d (双足落地, bipedal landing), 后肢先着 地并弯曲以进行缓冲, 主要发生在有垂直依附起跳 的飞跃后; YD16-e (四肢落地, quadrupedal landing), 四肢弯曲以进行缓冲，前后肢的触地时间略有间隔， 通常前肢先着地(Hunt et al, 1996)。

YD17 (树上摇摆, tree sway): 在树木间跨越间 隙的移动方式, 摇摆树以增加树枝的摆动幅度, 或 者利用体重以使树枝弯曲直至可以接触到另一棵 树。与桥渡不同，树上摇摆利用的是体重和摆动而 不是跃进(Hunt et al, 1996)。

YD18 (骑跨, ride): 与树上摇摆相似, 但该行为 是从树上到地面, 个体依附于一个垂直细小的树枝 上, 依靠摇摆或运动打破支撑物的平衡, 体重使得 树从垂直变成水平, 之后, 个体形成一个悬挂的动 作，随后后肢松开，脚掌先接触地面(Hunt et al, 1996)。

对灵长类肌肉骨骼系统的完全认识必须依赖 于一定数量的灵长类位置行为数据, 这样才能将行 为学和解剖学联系起来(Tuttle, 1969; Stern \& Oxnard, 1973; Jenkins \& Fleagle, 1975; Fleagle, 1979; Rodman, 1979)。但是, 位置行为的数据必须是详细 明确且定量的, 需要收集有关灵长类在其自然栖息 地的自然表现。所以, 位置行为的分类至关重要 (Hunt et al, 1996)。虽然不同物种的位置行为各有其 特殊性, 但是一些常见的行为模式是有共性的。在 对不同物种的研究中, 我们可参照位置行为的标准 化分类体系对该物种的行为模式进行更加详细具 体的定义, 从而减少在对灵长类各物种或不同地域 相同物种的位置行为比较中的误差。

然而, 也有一些学者认为, 很难建立一套可以 囊括并适用于体重相差很大的各个灵长类物种的 位置行为学标准体系(如从重 $35 \mathrm{~g}$ 、小鼠般大的狐猴 到 $180 \mathrm{~kg}$ 重的成年雄性大猩猩)(Garber, 2007)。不 同物种的四肢和身体比例差异显著。比如有无尾 巴、尾巴是否具抓握功能、有无对生的大拇指或无 功能的残留拇指等。此外, 历史上原本体型相同的 两种灵长类, 在进化过程中体型也会产生显著差异 (比如, 一种变大而另一种变小), 也会因异速生长
轨迹和不同的身体比例而产生不同的运动方式 (Garber, 2007)。所以, 一些重要的位置行为数据可 能会在这样的标准分类系统中丢失。

\section{2 影响位置行为的主要因素}

影响灵长类位置行为的潜在因素很多。位置行 为模式不仅取决于个体发育、体型大小和解剖学特 征, 还取决于非生物环境和生物环境的时空差异, 而栖息地的利用是位置行为模式的主要影响因素。

\section{1 季节性和具体地点的差异性}

研究发现, 在移动中行为模式具有显著的一致 性: 在进食地点间移动时, 个体可能会重复使用特 殊的树栖途径并选择安全有效的支撑物(Gebo \& Chapman, 1995a; Crompton, 1984; Dagosto, 1995; McGraw, 1998b; Garber, 1998)。灵长类位置行为在 季节性和具体地点两方面具显著差异。形成这种显 著差异的主要原因可能是进食、受食策略以及食物 供给和分配的季节性变化 (Grand，1972; CharlesDominique, 1977; Garber, 1980, 1984; Crompton, 1984; Cant, 1987a; Boinski, 1989; McGraw, 1998a)。

相同种类的灵长类在其栖息地的特殊位置行 为, 或者同一位置行为在不同栖息地的使用频率差 异, 均与它们对自然生境的适应性有关。Youlatos (2002)和 Mittermeier (1978)分别在法属圭亚那地区 和苏里南对两个红脸蜘蛛猴(Ateles paniscus)种群进 行了研究。其结果体现了蜘蛛猴属两个不同种群位 置行为的使用频率对于具体地点的差异性。在这两 个地点, 尾臂悬挂最为常见, 而跳跃和桥接最不常 见。两地点间最大的不同之处在于攀登/攀爬的频率 (在法属圭亚那地区频率为 $28.1 \%$, 而在苏里南地区 为 $17.0 \%$ )。而黑掌蜘蛛猴(Ateles geoffroyi) 位置行为 在具体地点间的差异性更为显著。Bergeson(1996) 用同一方法在两个不同地点(干燥的热带森林和湿 润的热带雨林)对黑掌蜘蛛猴进行研究并发现, 种 内在穿越沟壑时位置行为的显著不同表现为: 桥接 行为在较湿润的森林里明显多于在干燥的森林里, 尾臂悬挂行为明显较少, 而其他形式的位置行为使 用频率几乎相同。在 Dagosto (1995)的研究中发现原 猴类在不同季节和具体地点间移动时的行为模式 十分保守，而进食时的位置行为则存在很大差异。

\section{2 性别差异性}

由于雌性和雄性在体重、繁殖所需的营养消 耗、社会地位、获取资源方式, 以及成年雄、雌性 
所担任的对婴猴照顾和保护角色等方面的不同，一 般认为性别产生的位置行为差异性具有显著性。但 是, 在许多类群的灵长类中雄性与雌性在位置行为 上的差异极其细微。例如, 就普通黑猩猩来说(Pan troglodytes, 雄性比雌性重～30\%), Doran (1993)发 现, 无论是在进食还是移动时, 位置行为发生的频 率在两性间无显著差异。出现这一现象的部分原因 是雌、雄性黑猩猩的主要位置行为均为地面上的四 足行走。然而, Doran (1993)也阐述了雌、雄性黑猩 猩在树栖进食行为中的差异性, 雄性此时的攀爬行 为增加, 而雌性的四肢移动行为增加。但树栖的进 食行为在黑猩猩所有的位置行为中只占到 $2.2 \%$ 。因 此，黑猩猩因性别而产生的位置行为差异微小。

Cant (1987b)在对苏门答腊猩猩䧳、雄两性的位 置行为进行的研究发现, 雄性相比䧳性更趋向于利 用较大的支持物, 䧳性则出现更多的悬挂行为, 而 在进食中雄性则表现较多的坐和站立行为。猩猩是 最大的树栖哺乳动物, 且雄性和䧳性的体重相差很 大(雄性的体重几乎是雌性的 2.2 倍), 其位置行为的 差异也可能与其体重有关。Gebo \& Chapman (1995b) 在研究树栖狝猴时发现, 雌性和雄性在姿势行为和 移动行为以及对支持物的使用(尺寸和方向)等方面 几乎没有差异, 即使在高度二型(因性别不同而在 体型方面具有很大差异)的种类中也是如此。他们将 这一现象总结为, 不论是种间还是种内, 身体尺寸 的大小与位置行为的频率变化并无紧密联系(Gebo \& Chapman, 1995b)。

将猿类和旧大陆猴的研究数据一并考虑, 研究 者发现䧳、雄两性在移动行为、姿势行为和对支持 物的使用等方面无显著差异(Gebo, 1992; Gebo \& Chapman，1995b)。该结果支持灵长类物种(至少在 成年群体中)位置行为模式高度保守的观点。

从目前的研究结果来看, 位置行为的性别差异 在大多数灵长类物种中并不显著。然而也有研究发 现有些物种存在位置行为的性别差异, 且该差异似 乎更多的与社交关系和社群角色有关。比如, Chatani (2003) 发现成年雌、雄日本猴(Macaca fuscata) 个体在受食过程中的姿态行为存在差异, 且认为这些差异与其在形态、食物选择、社会活动 以及育幼行为中的投入有关。

\section{3 年龄差异性}

许多灵长类动物的行为模式随个体发育而变 化 (Garber, 2007; Prates \& Bicca-Marques, 2008)。研
究表明，体型大小、动作技能和生理状态的两性差 异以及个体发育是导致个体行为差异的重要因素 (Dunbar \& Badam, 1998; Chatani, 2003; Prates \& Bicca-Marques, 2008)。并且, 个体行为模式在不同 发育阶段也表现明显差异。Dunbar \& Badam (1998) 在对帽猴 (M. Radiata) 和长尾叶猴 (Presbytis entellus) 不同发育阶段的个体行为模式的比较研究中发现, 少年时期的姿势和移动行为最为丰富。其他研究则 表明四足行走频率随年龄增加而增加, 而跳跃频率 则相应减少(Bezanson, 2006; Prates \& Bicca-Marques, 2008)。

Li et al (2012)对半野生猕猴位置行为的性别年 龄差异的研究表明, 猕猴在活动高度、移动行为等 方面存在年龄差异性, 但无明显的性别差异。主要 表现为：成年个体在地面活动的时间明显多于亚成 年个体; 亚成年个体和少年个体在较高位置活动的 时间明显多于成年个体。即成年个体更偏好于在地 面活动，而亚成年个体和少年个体则花费更多的时 间在树上活动。在移动时, 成年个体更多采用四足 行走的移动模式，而亚成年个体则更倾向于采用跳 跃和架桥等移动模式。

灵长类动物姿势和移动行为的年龄差异性可 能与不同年龄阶段个体的体型、力量、协调性、技 能和活动水平差异相关(Prates \& Bicca-Marques, 2008)。

\section{4 其他因素}

对于陆地或树栖生活的生物, 环境对其体重、 四肢和身体比例的影响密切, 许多灵长类在世系进 化中形成了各自独特的适应性行为模式。研究表明, 原猴类和猿类的位置行为和支持物使用模式与其 各自的体重、受食策略以及形态方面的适应性紧密 相关(Fleagle \& Mittermeir, 1980)。这就需要重新评 估以体重为主要影响因素所预测的树栖环境下的 位置行为和食物资源利用模式。表 1 为生理因素、 生态因素及社会结构等方面对物种种内位置行为 多样性的影响 (Garber, 2007)。

\section{3 位置行为的发生及发展}

Dunbar \& Badam (1998)对恒河猴 Rhesus macaque)、帽猴(Bonnet macaque)、长尾叶猴 (hanuman langur)进行了研究, 描述了姿势和移动 行为的发展。他们将行为按照婴猴 1 期、婴猴 2 期、 青少年猴、成年猴、和老年猴的年龄组进行了讨论 
表 1 影响种内位置行为模式多样性的因素

Tab. 1 Factors influencing intraspecific variability of positional behavior patterns

\begin{tabular}{|c|c|c|}
\hline $\begin{array}{c}\text { 生理因素 } \\
\text { Physiological factors }\end{array}$ & $\begin{array}{c}\text { 生态因素 } \\
\text { Ecological factors }\end{array}$ & $\begin{array}{c}\text { 社交及行为影响 } \\
\text { Social and behavioral } \\
\text { factors }\end{array}$ \\
\hline $\begin{array}{c}\text { 体重及新陈代谢速率 } \\
\text { Body mass and } \\
\text { Metabolic rate }\end{array}$ & $\begin{array}{c}\text { 树冠的空间结构 } \\
\text { Three dimensional } \\
\text { structure of arboreal } \\
\text { canopy } \\
\end{array}$ & $\begin{array}{l}\text { 种群大小 } \\
\text { Group size }\end{array}$ \\
\hline $\begin{array}{c}\text { 两性异形 } \\
\text { Sexual dimorphism } \\
\end{array}$ & $\begin{array}{c}\text { 栖息地类型 } \\
\text { Forest type } \\
\end{array}$ & $\begin{array}{c}\text { 食物竞争 } \\
\text { Feeding competition } \\
\end{array}$ \\
\hline $\begin{array}{c}\text { 位置行为的能量消耗 } \\
\text { Energetic cost of } \\
\text { positional behaviors }\end{array}$ & $\begin{array}{c}\text { 支撑物类型与食物供给 } \\
\text { 分配的空间关系 } \\
\text { Spatial relationship } \\
\text { between support }\end{array}$ & $\begin{array}{c}\text { 亚群的类型及社群间隔 } \\
\text { Patterns of subgrouping } \\
\text { and social spacing }\end{array}$ \\
\hline $\begin{array}{c}\text { 个体发育时体重以及四 } \\
\text { 肢和身体比例的改变 } \\
\text { Ontogenetic changes in } \\
\text { body weight and limb } \\
\text { and body proportions }\end{array}$ & $\begin{array}{c}\text { 食物供给分配的 } \\
\text { 季节性变化 } \\
\text { Seasonal changes in } \\
\text { food available and } \\
\text { distribution }\end{array}$ & $\begin{array}{c}\text { 种群角色和行为 } \\
\text { 模式的不同 } \\
\text { Differences in social role } \\
\text { and activity pattern }\end{array}$ \\
\hline $\begin{array}{c}\text { 繁殖条件 } \\
\text { Reproductive condition }\end{array}$ & $\begin{array}{c}\text { 栖息地模式的性别年龄 } \\
\text { 差异 } \\
\text { Age or sex based } \\
\text { differences in patterns of } \\
\text { habitat utilization }\end{array}$ & $\begin{array}{c}\text { 等级 } \\
\text { Dominance }\end{array}$ \\
\hline $\begin{array}{c}\text { 营养需求 } \\
\text { Nutritional requirements }\end{array}$ & $\begin{array}{l}\text { 对掠食者的躲避 } \\
\text { Predator avoidance }\end{array}$ & $\begin{array}{c}\text { 联盟关系或亲属关系 } \\
\text { Affiliative bonds and } \\
\text { kinship } \\
\end{array}$ \\
\hline $\begin{array}{c}\text { 温度调节 } \\
\text { Thermoregulation }\end{array}$ & & $\begin{array}{c}\text { 行为的个体特异性和年 } \\
\text { 龄及性别方面的不同 } \\
\text { Idiosyncratic, age, and } \\
\text { sex-based differences in } \\
\text { behavior }\end{array}$ \\
\hline
\end{tabular}

(表 2)。贞猴 1 期用手脚强有力地抓握依附在母猴腹 部。独立的协调运动行为发展于紧猴 2 期。青少年 时期位置行为的定性、定量发展达到最高水平。成 年期和老年期运动能力的多样性及质量逐步下降。 但是，猕猴和叶猴的行为发展过程有所不同。这些 发现对神经机制的概念产生了影响, 表明这些机制 的发展可能存在物种差异性。

三个物种的新生婴猴均不能独立行走、站立或

表 2 不同灵长类物种年龄组划分

Tab. 2 Approximate chronological ages for groups in each species

\begin{tabular}{cccc}
\hline 年龄组 & $\begin{array}{c}\text { 恒河猴 } \\
\text { Age group }\end{array}$ & $\begin{array}{c}\text { 帽猴 } \\
\text { Macaca mulatta }\end{array}$ & $\begin{array}{c}\text { Macaca 长尾叶猴 } \\
\text { Presbytis entellus }\end{array}$ \\
\hline 婴猴 1 期 & $0 \sim 2$ 个月 & $0 \sim 2$ 个月 & $0 \sim 3$ 或 5 个月 \\
Infant-1 & $0 \sim 2$ months & $0 \sim 2$ months & $0 \sim 3$ or 5 months \\
贞猴 2 期 & $2 \sim 12$ 个月 & $2 \sim 12$ 个月 & 3 或 5 个月 15 个 \\
Infant- 2 & $2 \sim 12$ months & $2 \sim 12$ months & 月 \\
青少年猴 & $1 \sim 3$ 或 4 岁 & $1 \sim 3$ 或 4 岁 & 15 个月 $\sim 4$ 岁 \\
Juvenile & $1 \sim 3$ or 4 years & $1 \sim 3$ or 4 years & 15 months $\sim 4$ years \\
成年猴 & 3 或 4 15 岁 & 3 或 4 15 岁 & $4 \sim 15$ 岁 \\
Adult & 3 or 4 15 years & 3 or $4 \sim 15$ years & $4 \sim 15$ years \\
老年猴 & $15 \sim 20$ 岁及以上 & $15 \sim 20$ 岁及以上 & $15 \sim 20$ 岁及以上 \\
Elderly & $15 \sim 20+$ years & $15 \sim 20+$ years & $15 \sim 20+$ years \\
\hline
\end{tabular}

端坐。在贞猴 1 期的后期开始出现一些短暂的移动 行为(如，四足行走、双足跳跃), 但肢体运动显著不 协调, 并且在节平面(pitch plane)头部会过度旋转或 上下摆动。处于婴猴 2 期的恒河猴、帽猴, 和肾猴 2 期后期的长尾叶猴已经出现了大多数成年猴的基 本姿势和移动行为。独立姿势和移动行为的出现是 㚣猴 1 期到贞猴 2 期过渡的一项行为指标。虽然在 这段时间内紧猴的动作越来越独立, 但它们与其母 亲的距离仍然保持在 $1 \mathrm{~m}$ 范围内。最初其动作显得 相对笨拙和不协调, 表现在: 婴猴经常会绊倒、踩 空或失去平衡(特别是在树上)。皮质神经连接 (corticomotoneuronal connections) 在出生后第三个 月末就已经全面建立了, 这使得婴猴 2 期个体能够 精确顺利地释放抓握物品, 以及进行相对独立的手 指动作 (relatively independent finger movements, RIFM)(Lawrence \& Hopkin, 1976), 其行为也越来越 细化。在这三个灵长类物种中, 青少年时期是位置 行为发展的黄金时期。与成年猴相比较灵活的体型 与已近乎完善的力量和协调性相结合 (Dunbar, 1994), 使得青少年猴能完全利用陆地和树木资源 进行受食、玩要和移动。青少年时期的主要特征是 其位置行为大多发生在小型树枝末端(Dunbar, 1989)。在树枝上的姿势行为包括仰卧、俯卧、2 4 只前后肢站立; 在树枝下的悬挂行为包括头向上的 前肢悬挂和头可伸向各方向的前后肢悬挂。青少年 猴在地面和较大直径的树枝上的行走步态通常为 对角线行走，而当作为支撑物的树枝直径逐渐减小 时, 青少年猴就会转变为非对称步态行走(YD1-b), 从而减少其失去平衡而从树枝上掉下的可能性 (Gray, 1944)。青少年猴在树木上的奔跑也仅限于在 较大直径的树枝上。除了在玩要外(这时它们常常会 扭转四肢), 青少年猴在飞跃过程中身体是十分僵 硬的(Dunbar, 1989), 为了减少受伤的危险, 整个身 体的扭转被控制在最少(Dunbar, 1988, 1994)。Napier \& Walker (1967)首先在原猴类中观察到垂直依附型 飞跃, 研究人员随后对其进行了生物力学分析 (Dunbar, 1988, 1994; Peters \& Preuschoft, 1984)。结 果表明, 这是青少年猴(和较年长的紧猴 2 期猴)进 行的运动学上非常复杂的一种跳跃方式, 并且在成 年猴中尚未发现。这些随年龄增长而出现的行为变 化可能更多地与身体比例的变化和重量分布有关, 而非受神经系统控制(Dunbar, 1994)。成年猴与青少 年猴相比, 其姿势和移动行为总数减少, 而不是如 
人们一般所认为的增多。减少最多的行为是青少年 猴在小树枝上的玩要行为, 并且在树枝下进行的姿 势和移动行为均消失。成年长尾叶猴常常会做出一 些惊人并多样的树木间跳跃(Rosenblum，1968)。相 反, 成年狝猴只会在必要时偶尔小心翼翼地进行一 些简单的短距离跳跃。对于这 3 个物种来说, 成年 猴在陆地上的移动行为包括: 行走、奔跑和跳跃 (Grand, 1976)。与在青少年猴中的观察相同(Dunbar \& Badam, 1995), 不同的移动行为模式会有不同的 头部和躯干的动作。在行走时, 头可在躯干上转动, 而在急速奔跑和跳跃式时, 身干会绕头部而转动。 老年猴的位置行为模式相对成年猴减少，且存在本 质上的区别。老年猴往往坐和躺的动作较多而不常 移动 (Rawlins, 1976), 尽可能的避免奔跑、跳跃和 攀爬, 且在树枝上的姿势和移动行为也仅限于最大 的树枝。这些行为上的限制主要反映了机械力学的 变化和衰退(如关节机动性的限制，骨骼的退化以 及视力调节的丧失)(Derousseau et al，1986; Turnquist, 1986; Pyykkö et al, 1988), 而非神经系统 的变化。

目前对于其他灵长类物种位置行为的发生与 发展尚未有全面系统的研究。此方面的研究对于认 识灵长类进化史有一定的意义, 并有助于进行物种 划分及辨别。

\section{4 结 语}

目前, 灵长类位置行为的研究主要关注于姿势 行为和移动行为的区分、位置行为分类的定义、以 及灵长类尾巴是起到平衡还是重力支持作用的区 分、体型大小对支持点的偏好性选择和对位置行为 的影响、以及季节性和具体地点、不同性别年龄组 的位置行为的差异性等。

Hunt et al 对位置行为的标准化定义已近乎全 面, 但由于栖息地的潜在变化和灵长类的物种多样 性, 可能还会不断有新的位置行为模式被发现并记

\section{参考文献:}

Anemone RL. 1993. The function anatomy of the hip and thigh in primates [C] // Gebo DL. Postcranial Adaptation in Nonhuman Primates. Dekalb: Northern Illinois University Press, 150-174.

Bergeson DJ. 1996. The Positional Behavior and Prehensile Tail Use of Alouatta palliate, Ateles geoffroyi, and Cebus capucinus [D]. Ph.D. Dissertation, Washington University, St. Louis, Missouri.

Bergeson DJ. 1998. Patterns of suspensory feeding in Alouatta palliata,
录。在对非人灵长类动物位置行为的研究更加成熟 之后, 还可比较非人灵长类和人类之间的姿势和运 动行为发生和发展的异同。对于具体野生个体的位 置行为研究还受限于个体识别, 尤其是青少年猴及 亚成年猴，它们一般难以进行个体区分，并且其位 置行为十分多样，数据记录也有一定的困难。随着 科技水平的进步和对野生猴群的研究深入以及国 内外学者们对位置行为研究的关注, 位置行为的研 究将会有进一步的发展。

中国是全球少数几个灵长类动物资源最丰富 的国家之一, 在现存的 200 余种灵长类动物中, 中 国分布有 3 科(懒猴科、猴科、长臂猿科) 20 余种、 40 47 个亚种, 主要包括蜂猴属、狝猴属、长尾叶 猴属、乌叶猴属、仰鼻猴属、长臂猿属等。其中川 金丝猴 (Rhinopithecus roxellanae)、滇金丝猴 (Rhinopithecus bieti)、黔金丝猴 (Rhinopithecus brelichi)、藏酋猴(Macaca thibetana)及台湾猴为我国 特有种。黑长臂猿(Hylobates concolor)、白掌长臂 猿(Hylobates lar)、白眉长臂猿(Hylobates hoolock) 及海南长臂猿等至少 14 个亚种濒临灭绝(Pan \& Kun, 1984), 而白臀叶猴(Pygathris nemaeus) 已在我 国绝迹(或有争议)。不同物种根据生态位的不同进 化出适应不同生境的位置行为模式。目前我国灵长 类研究主要涉及的领域为生态学、遗传学、生理学、 医学、社会行为学等方面, 而野生灵长类位置行为 的研究近乎空白。然而此方面的研究对于理解灵长 类适应性的多样化、灵长类生态学、解剖学以及灵 长类的进化史十分必要, 并将对中国灵长类动物资 源的保护产生重要意义。

致谢: 美国伊利诺伊斯州立大学 Paul A. Garber 教授, 西北大学朱文文、黄康、张东、付卫玮、张 红阳、吴林林、李娟娟、侯立、王荣涛、侯㭉诸君 在本文执笔和文献查阅中给予无私帮助，在此谨致 衰心感谢。
Ateles geoffroyi, and Cebus capucinus [C] // Strasser E, Fleagle JG, Rosenberger A, McHenry H. Primate Locomotion: Recent Advances. New York: Plenum Press, 45-60.

Bezanson MF. 2006. Leap, bridge, or ride? Ontogenetic influences on positional behavior in Cebus and Alouatta [C] // Estrada A, Garber PA, Pavelka MSM, Luecke L. New Perspectives in the Study of Mesoamerican Primates: Distribution, Ecology, Behavior, and 
Conservation. New York: Springer Verlang, 333-348.

Boinski S. 1989. The positional behavior and substrate use of squirrel monkeys: Ecological implications [J]. J Hum Evol, 18(7): 659-677.

Cant JGH. 1986. Locomotion and feeding posture of spider and howling monkeys: field study and evolutionary interpretation [J]. Folia Primatol, 46(1): 1-14.

Cant JGH. 1987a. Positional behavior of female bornean orangutans (Pongo pygmaeus) [J]. Am J Primatol, 12(1): 71-90.

Cant JGH. 1987b. Effects of sexual dimorphism in body size on feeding postural behavior of Sumatran orangutans (Pongo pygmaeus) [J]. Am J Phys Anthropol, 74(2): 143-148.

Charles-Dominique P. 1977. Ecology and Behavior of Nocturnal Primates [M]. New York: Columbia University Press.

Chatani K. 2003. Positional behavior of free-ranging Japanese macaques (Macaca fuscata) [J]. Primates, 44(1): 13-23.

Crompton RH. 1984. Foraging, habitat structure, and locomotion in two species of Galago [C] // Rodman PS, Cant JGH. Adaptations for Foraging in Nonhuman Primates. New York: Columbia University Press, 73-111.

Dagosto M. 1995. Seasonal variation in positional behavior of Malagasy lemurs [J]. Int J Primatol, 16(5): 807-834.

Derousseau CJ, Bito LZ, Kaufman PL. 1986. Age-dependent impairments of the rhesus monkey visual and musculoskeletal systems and apparent behavioral consequences [C] // Rawlins RG, Kessler MJ. The Cayo Santiago Macaques. Albany: State University of New York Press, 233-251.

Doran DM. 1993. Sex differences in adult chimpanzee positional behavior: the influence of body size on locomotion and posture [J]. Am J Primatol, 91(1): 99-116.

Dunbar DC, Badam GL. 1995. Head and trunk stabilization in natural gaits of free-ranging monkeys [J]. Soc Neurosci Abstr, 21: 418.

Dunbar DC, Badam GL. 1998. Development of posture and locomotion in free-ranging primates [J]. Neurosci Biobehav Rev, 22(4): 541-546. Dunbar DC. 1988. Aerial maneuvers of leaping lemurs: the physics of whole-body rotations while airborne [J]. Am J Primatol, 16(4): 291-303.

Dunbar DC. 1989. Locomotor behavior of rhesus macaques (Macaca mulatta) on Cayo Santiago [J]. P R Health Sci J, 8(1): 79-85.

Dunbar DC. 1994. The influence of segmental movements and design on whole-body rotations during the airborne phase of primate leaps $[\mathrm{J}] . Z$ Morphol Anthropol, 80(1): 109-124.

Fleagle JG. 1978. Locomotion, posture and habitat utilization in two sympatric Malayan leaf monkeys [C] // Montogomery GE. Ecology of Arboreal Folivores. Washington DC: Smithsonian Press, 243-251.

Fleagle JG. 1979. Primate positional behavior and anatomy: naturalistic and experimental approaches [C] // Morbeck ME, Preuschoft H, Gomberg N. Environment, Behavior and Morphology: Dynamic Interactions in Primates. New York: Gustav Fischer Verlag, 313-325.

Fleagle JG, Mittermeier RA. 1980. Locomotor behavior, body size, and comparative ecology of seven Surinam monkeys [J]. Am J Phys Anthropol, 52(3): 301-314.

Fontaine R. 1990. Positional behavior in Saimiri boliviensis and Ateles geoffroyi [J]. Amer J Phys Anthropol, 82(4): 485-508.

Garber PA. 1980. Locomotor behavior and feeding ecology of the Panamanian tamarin (Saguinus oedipus geoffroyi, Callitrichidae, Primates) [J]. Int J Primatol, 1(2): 185-210.
Garber PA. 1984. Use of habitat and positional behavior in a neotropical primates, Saguinus Oedipus [C] // Rodman PS, Cant JGH. Adaptations for Foraging in Nonhuman Primates: Contributions to an Organismal Biology of Prosimians, Monkeys, and Apes. New York: Columbia University Press, 112-133.

Garber PA. 1992. Vertical clinging, small body size, and the evolution of feeding adaptation in the Callitrichinae [J]. Am J Phys Anthropol, 88(4): 469-482.

Garber PA. 1998. Within- and between-site variability in moustached tamarin (Saguinus mystax) positional behavior during food procurement [C] // Strasser E, Fleagle JG, Rosenberger A, McHenry H. Primate Locomotion: Recent Advances. New York: Plenum Press, 61-78.

Garber PA. 2007. Primate locomotor behavior and ecology [C] // Campbell CJ, Fuentes A, MacKinnon KC, Panger M, Bearder SK. Primates in Perspective. New York: Oxford University Press, 543-560.

Gebo DL, Chapman CA. 1995a. Positional behavior in five sympatric old world monkeys [J]. Am J Phys Anthropol, 97(1): 49-76.

Gebo DL, Chapman CA. 1995b. Habitat, annual, and seasonal effects on positional behavior in red colobus monkeys [J]. Am J Phys Anthropol, 96(1): 73-82.

Grand TI. 1972. A mechanical interpretation of terminal branch feeding [J]. J Mammal, 53(1): 198-201.

Grand TI. 1976. Differences in terrestrial velocity in Macaca and Presbytis [J]. Am J Phys Anthropol, 45(1): 101-108.

Gray J. 1944. Studies in the mechanics of the tetrapod skeleton [J]. $J$ Exp Biol, 20(2): 88-116.

Hunt KD, Cant JGH, Gebo DL, Rose MD, Walker SE, Youlatos D. 1996. Standardized descriptions of primate locomotor and postural modes [J]. Primates, 37(4): 363-387.

Hunt KD. 1991. Positional behavior in the Hominoidea [J]. Int J Primatol, 12(2): 95-118.

Jenkins FA Jr, Fleagle JG. 1975. Knuckle-walking and the functional anatomy of the wrists in living apes [C] // Tuttle RH. The Functional and Evolutionary Biology of Primates. Chicago: Aldine, 213-228.

Jiang ZG. 2004. Theories in Animal Behavioral Study and Conservation Methods [M]. Beijing: Science Press. [蒋志刚. 2004. 动物行为原理 与物种保护方法. 北京: 科学出版社.]

Kinzey WG. 1967. Preface [J]. Am J Phys Anthropol, 26(2): 115-118.

Lawrence DG, Hopkins DA. 1976. The development of motor control in the rhesus monkey: evidence concerning the role of corticomotoneuronal connections [J]. Brain, 99(2): 235-254.

Li DY, Zhou QH, Tang HX, Huang CM. 2012. Sex-age differences in activity budget and position behavior of rhesus macaques (Macaca mulatta) [J]. Acta Ther Sin, 32(1): 25-32. [黎大勇, 周崎海, 唐兴华, 黄乘明. 2012. 狝猴不同性别年龄组个体时间分配和姿态行为的差 异分析. 兽类学报, 32(1): 25-32.]

McGraw WS. 1998a. Posture and support use of Old World monkeys (Cercopithecidae): the influence of foraging strategies, activity patterns, and the spatial distribution of preferred food items [J]. Am J Primatol, 46(3): 229-250.

McGraw WS. 1998b. Locomotion, support use, maintenance activities, and habitat structure: the case of the Ta ï Forest cercopithecids [C] // Strasser E, Fleagle JG, Rosenberger A, McHenry H. Primate Locomotion: Recent Advances. New York: Plenum Press, 79-94.

Mittermeier RA. 1978. Locomotion and posture in Ateles geoffroyi and 
Ateles paniscus [J]. Folia Primatol, 30(3): 161-193.

Napier JR, Walker AC. 1967. Vertical clinging and leaping-a newly recognized category of locomotor behaviour of primates $[\mathrm{J}]$. Folia Primatol, 6(3-4): 204-219.

Niemitz C. 1984. Locomotion and posture of Tarsius bancanus [C] // Niemitz C. Biology of Tarsiers. New York: Gustav Fischer Verlag, 191-226.

Oxnard CE, Crompton RH, Liebermann SS. 1990. Animal Lifestyles and Anatomies: The Case of the Prosimian Primates [M]. Seattle: Washington University Press.

Pan QH, Ben KL. 1984. The review and prospect of primatology in China [J]. Zool Res, 5(S4): 1-6. [潘清华, 贲昆龙. 1984. 中国灵长类研究 工作的回顾与展望. 动物学研究, 5(S4): 1-6.]

Peters A, Preuschoft H. 1984. External biomechanics of leaping in Tarsius and its morphological and kinematic consequences [C] // Niemitz C. Biology of Tarsiers. New York: Gustav Fischer Verlag, 227-255.

Prates HM, Bicca-Marques JC. 2008. Age-sex analysis of activity budget, diet, and positional behavior in Alouatta caraya in an orchard forest [J]. Int J Primatol, 29(3): 703-705.

Prost JH. 1965. A definitional system for the classification of primate locomotion [J]. Am Anthropol, 67(5): 1198-1214.

Pyykkö I, Jäntti P, Aalto H. 1988. Postural control in the oldest olds [J]. $A d v$
Otorhinolaryngol, 41: 146-151.

Rawlins RG. 1976. Locomotor ontogeny in Macaca mulatta: I. Behavioral strategies and tactics [J]. Am J Phys Anthropol, 44: 201.

Ripley S. 1967. The leaping of langurs: a problem in the study of locomotor adaptation [J]. Am J Phys Anthropol, 26(2): 149-170.

Rodman PS. 1979. Skeletal differentiation of Macaca fascicularis and Macaca nemestrina in relation to arboreal and terrestrial quadrupedalism [J]. Am J Phys Anthropol, 51(1): 51-62.

Rosenblum LA. 1986. Mother-infant relations and early behavioral development in the squirrel monkey [C] // Rosenblum L, Cooper R. The Squirrel Monkey. New York: Academic Press, 207-233.

Stern JT Jr, Oxnard CE. 1973. Primate locomotion: some links with evolution and morphology [J]. Primatologia, 4(11): 1-93.

Turnquist JE. 1986. Joint mobility as a function of age in free-ranging rhesus monkeys (Macaca mulatta) [C] // Rawlins RG, Kessler MJ. The Cayo Santiago Macaques. Albany: State University of New York Press, 253-262.

Tuttle RH. 1969. Quantitative and functional studies on the hands of the anthropoidea. I: the Hominoidea [J]. J Morphol, 128(3): 309-363.

Youlatos D. 2002. Positional behavior of black spider monkeys (Ateles paniscus) in French Guiana [J]. Int J Primatol, 23(5): 1071-1093.

\section{秦岭川金丝猴研究中心简介}

该团队自 2000 年来，在国家自然科学基金的持续资助下，对秦岭川金丝猴进行了长期而深入的研究。 取得以下重要成果：1）经过对秦岭川金丝猴种群的分布和数量的系统调查，结合对 400 年来中国金丝猴 群分布变迁的分析，揭示了秦岭成为它们最后的避难所，并且仍受到生境破碎化的威胁。2）对秦岭川金 丝猴的栖息地选择、家域利用、食性和种群参数进行了综合研究, 揭示了它们泛化的食性、大的家域特征, 阐明了这类本应在热带亚热带生存的叶猴种类对寒温带气候的适应机制。3）在研究交配选择和个体扩散 机制的基础上，首次发现这个物种的交配制度是混交制。提出了在破碎化生境斑块间建立走廊带，使得孤 立种群间个体得以沟通，恢复基因交流，解决了种群隔离面临的实际保护问题。4）通过“招引性投食”的方 法, 使得树栖灵长类可以被近距离观察并获得成功, 实现了“个体识别”, 满足了个体行为和社会关系定量 分析的条件, 为我国灵长类动物与大型哺乳动物的行为学研究提供了可借鉴的示范样板。5）运用个体识 别的手段, 对野生种群的个体行为和社会关系进行系统研究, 发现这种大型的社群具有分层的社会结构和 严密的组织形式, 阐明了它们的社会结构、等级制度、交配选择和行为发育过程, 初步揭开了川金丝猴社 会体系的神秘面纱一一一夫多妻制的松散母系社会。推动了中国灵长类行为生态学研究的发展。6）建立 了“中国第一个金丝猴野外研究基地”, 为野生动物种群的长期监测和保护研究开创了新的道路。该团队曾 得到 5 项国家自然科学基金资助。发表相关论文百余篇, 其中 SCI 收录近 50 篇。国际灵长类学会主办的 International Journal of Primatology 和美国灵长类学会主办的American Journal of Primatology 代表国际灵长 类研究的最高水平, 国际保护生物学会主办的 Conservation Biology 代表着国际保护生物学研究的最前沿。 该团队在这三大国际刊物上发表了相关论文 10 余篇。其的研究成果得到了国内外学术界的广泛关注与好 评, 在世界范围为我国的灵长类研究赢得了一席之地。 